\title{
Flame cooling by a burner wall
}

\author{
L. P. H. DE GOEY and H. C. DE LANGE \\ Faculty of Mechanical Engineering, WH.3-138, Eindhoven University of Technology, P.O. Box 513, \\ 5600 MB Eindhoven, The Netherlands
}

(Received 28 August 1992 and in final form 5 August 1993)

\begin{abstract}
A model is proposed to describe the structure of the cooling boundary layer between a cold burner wall and a flame near fiash-back. Two-dimensional combustion equations are solved using a onestep chemical reaction model. The analytical solutions prove to be in good agreement with the results of a numerical study. Furthermore, the thickness of the cooling layer is estimated and appears to be in fair agreement with available experimental data. The understanding of the mass and heat transport processes in a flame near a cold burner wall is indispensable for the understanding of the flame stabilization process.
\end{abstract}

\section{INTRODUCTION}

The STABILIZATION process of a premixed laminar flame near a cooled burner is determined by the transport processes in three boundary layers: the thermal, the diffusive and the shear boundary layers. The thickness of the thermal boundary layer, i.e. the horizontal distance between the flame and the burner wall, will be referred to as the stand-off distance $\delta_{\mathrm{q}}$. Roughly speaking, a flame stabilizes in such a way that the gas speed in the attachment point is equal to the adiabatic flame speed, as is shown in Fig. 1, schematically. This interpretation is used for the introduction of the wellknown critical gradients [1] to obtain a geometryindependent measure for blow-off and flash-back.

The stand-off distance changes with the level of the flame relative to the top of the burner. This dependency is caused by changes in the cooling rate by the burner wall and by diffusion of fuel in the surrounding atmosphere. The influence of cooling grows as the flame moves downwards, thus causing a local reduction of the flame speed and an increase of the stand-off distance. The influence of diffusion of fuel in the surrounding atmosphere increases if the flame is lifted further above the burner, leading to a reduction of the equivalence ratio in the gas flow. This decrease of the equivalence ratio causes an increase of the local value of the flame speed in fuel-rich flames and, thus, to a decrease of the stand-off distance. The decrease of the equivalence ratio has the opposite effect in fuel-lean flames.

The starting-point of this view of the stabilization

$†$ This description of the stabilization process does not account for the disruption of chain reactions in the flame by the diffusion of radicals towards the cold burner wall, which might very well be important in a more detailed description of flame stabilization [4].

\$The flame temperature, and consequently also the flame speed, is influenced mainly by the energy balance in the upstream part of the flame. principle of a flame is that it is linked directly with the local value of the flame speed $[2,3]$. The flame speed in its turn depends on the local values of the temperature and mass fractions. In this approach a description of the shape of the temperature and mass fractions profiles as a function of the position of the flame relative to the burner is indispensable for the understanding of the stabilization process. $†$ The main subject of this paper is the analysis of these profiles in the region between a cooled burner wall and a flame stabilized near the top of the wall. This is a flame at the flash-back limit, since the shape of the upstream boundary layers and, therefore, the local value of the flame speed, does not change any more when the flame moves further in the burner.

It is impossible to give an analytical solution of this problem in its full complexity. Therefore, a simplified model is proposed in which the velocity component perpendicular to the wall is assumed to be zero. Furthermore, the parallel velocity component at the

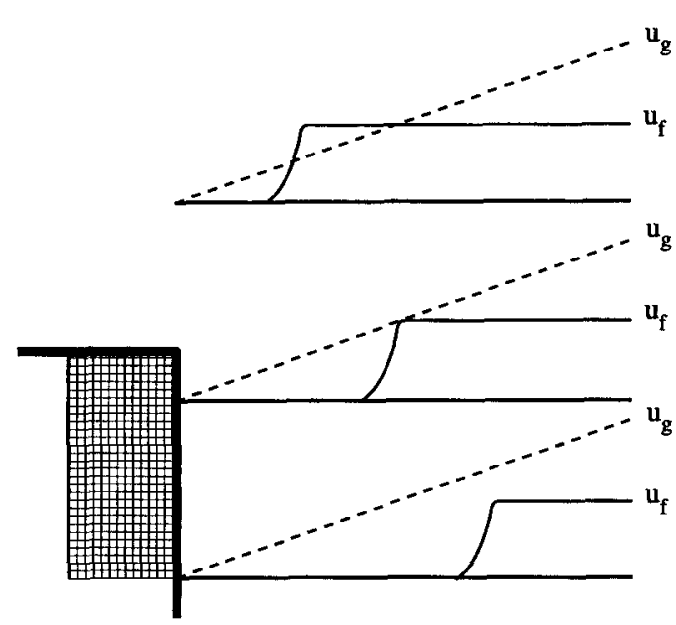

FIG. 1. The schematic velocity profiles and the flame speed at different levels near a cooled wall from the top downwards : the flame moves down, is stabilized and moves up. 


\section{NOMENCLATURE}

A reaction rate parameter $\left[\left(\mathrm{kg} \mathrm{m}^{-3}\right)^{i} x^{x-i}\right] \quad Y_{i} \quad$ mass fraction of species $i$.

$c_{\mathrm{p}}$ specific heat $\left[\mathrm{J} \mathrm{kg}^{-1} \mathrm{~K}^{-1}\right]$

$g$ the gradient of the velocity profile at the wall $\left[\mathrm{s}^{-1}{ }^{1}\right]$

$J_{i} \quad$ the Shvab-Zel'dovich variable for species $i$

$L \quad$ length scale $[\mathrm{m}]$

$L e_{i}$ the Lewis number of species $i$

$P \quad$ the pressure $\left[\mathrm{N} \mathrm{m}^{-2}\right]$

$R_{\mathrm{g}} \quad$ the specific gas constant $\left[\mathrm{J} \mathrm{kg}^{-1} \mathrm{~K}^{-1}\right]$

$s_{i} \quad$ the mass of species $i$ consumed per unit mass of fuel $\left[\mathrm{kg}_{i} \mathrm{~kg}_{\mathrm{fu}}^{-1}\right]$

$T$ temperature [K]

$T_{\mathrm{a}}$ reaction rate parameter $[\mathrm{K}]$

$u \quad x$-component of the velocity vector $\left[\mathrm{ms}{ }^{\prime}\right]$

v $y$-component of the velocity vector $\left[\mathrm{m} \mathrm{s}^{-1}\right.$

v velocity vector

Greck symbols

$\alpha$ reaction rate parameter

$\beta \quad$ reaction rate parameter

$\delta_{i} \quad$ length scale of the stand-off distance [m]

$\Delta h$ the amount of heat produced by the reaction $\left[\mathrm{J} \mathrm{kg}^{-1}\right]$

$\eta \quad$ length scale of the cooling layer $[\mathrm{m}]$

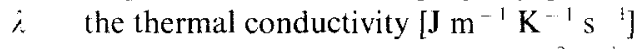

$\dot{\rho}_{\text {lu }} \quad$ fuel mass consumption rate $\left[\mathrm{kg} \mathrm{m}^{-3} \mathrm{~s}^{1}\right]$

$\rho \quad$ mixture density $\left[\mathrm{kg} \mathrm{m}^{-3}\right]$.

Superscripts

u the unburnt boundary conditions

b the burnt boundary conditions. inflow boundary is taken to be independent of the distance from the burner wall (as shown in Fig. 2). The gas speed has to be chosen equal to the adiabatic flame speed to find a stabilized flame in this situation.

The upstream part of a flat flame, propagating through a lube in the frame of reference connected to the flame, may be regarded as an experimental equivalent of the modeled system, at least as long as the post-flame zone has a negligible influence on the structure of the pre-heating zone. Actually, the model can be applied to any combustion system in which the shear boundary layer is much thinner than the thermal and diffusion boundary layers. Unfortunately, these boundary layers build up equally fast, since the Prandtl and Lewis numbers of gases are of order unity. Therefore, it seems impossible to create an equivalent flow situation in a stabilized flame, experimentally. However, the restriction imposed on the velocity field is necessary to derive analytical expressions for the structure of the thermal and diffusive boundary layers. The influence of the shape of the velocity profile will be studied to some extent in Appendix B, numerically.

The model presented in this paper is an extension of the study presented in ref. [5], where the same approximation for the velocity field is used. Additionally, the influence of lateral mass diffusion on the

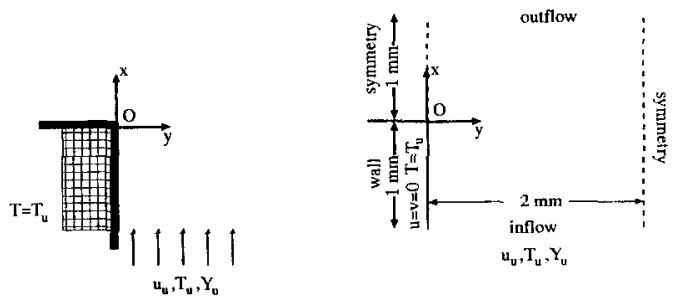

Fig. 2. The theoretical (left) and numerical (right) studied 2-D geometry. structure of the boundary layers is neglected in ref. [5]. The study presented in this paper shows that the balance of the lateral thermal and diffusive fluxes plays an important role in the principle of flame stabilization. Another approach is found in ref. [6], where the same restriction is used for the velocity field. They analyze the structure of the boundary layer for the case that conduction parallel to the wall is negligible as compared to conduction towards the wall. This description concentrates on cooling by the burner wall in the downstream part of the flame, whereas our description is concerned with the behavior of the boundary layers in the upstream part of the flame. Most of the other theoretical models (e.g. $[7,8]$ ) of cooled flames use an artificial heat-loss term in the energy equation.

The starting points of the study presented here, are the combustion equations as presented in Section 2 . The relation between the shape of the temperature and mass-fractions profiles near the cold burner wall is described schematically in Section 3. Expressions for the profiles of the temperature, mass fractions and the Shvab-Zel'dovich variables are derived in Sections 4,5 and 6, respectively. Furthermore, the thickness of the boundary layers is estimated in Section 8.

Along the way the analytical results will be confronted with the results of a two-dimensional numerical study of a stoichiometric $\mathrm{CH}_{4} /$ air flame, to support the simplifications made during the analysis. The numerical algorithm, which has been used, is described in refs. [9-11]. The numerical results arc obtained by using the same physical simplifications as made in the analytical model (constant thermal conductivity, constant specific heat and the onedimensional flow approximation). However, the mathematical simplifications introduced in the analytical description (e.g. the assumption of an infinitely 
thin reaction zone and the boundary conditions on the boundary of the cooling layer) are omitted in the numerical model. The numerical results are calculated on an cquidistant $81 \times 81$ grid on the geometry shown in Fig. 2. The chosen physical and chemical parameters, introduced in Section 2, lead to an adiabatic flame speed of $0.41 \mathrm{~m} \mathrm{~s}^{-1}$ for this flame. To find a stabilized flame numerically, the gas speed at inflow is taken to be equal to $0.4 \mathrm{~m} \mathrm{~s}^{-1}$. The flame is stabilized at the top of the burner by making the fuel consumption rate equal to zero for $x \leqslant-0.2 \mathrm{~mm}$. This places the maximum of the fuel consumption rate (i.e. the 'flame') approximately at the same level as the burner top.

The analytical results are confronted with some experimental results found in the literature in the discussion presented at the end of this paper (Section 9). In spite of the simplifications that have been made to obtain the analytical results, they compare reasonably well with experimental data. The influence of some of the simplifications, especially those concerning the thermal conductivity and the shape of the velocity profile (constant, linear and parabolic) are studied numerically in Appendices A and B.

\section{COMBUSTION EQUATIONS}

The combustion process is supposed to be a onestep irreversible reaction:

$$
\text { Fuel (fu) + Oxygen (ox) } \Rightarrow \text { Product (pr) }
$$

with an Arrhenius-like fuel mass consumption rate equal to $[12,13]$ :

$$
\dot{\rho}_{\mathrm{fu}}=-A \rho^{p}\left[Y_{\mathrm{fu}}\right]^{\alpha}\left[Y_{\mathrm{ox}}\right]^{\beta} \mathrm{e}^{\left(-T_{\mathrm{a}} / T\right)},
$$

where $\rho$ is the mixture density, $T$ is the temperature and $Y_{\mathrm{fu}, \mathrm{ox}}$ are the mass fractions of fuel and oxygen. Furthermore, $A, \alpha, \beta, p(=\alpha+\beta)$ and $T_{\mathrm{a}}$ are rate parameters. To obtain a physically realistic flame we have chosen a combination of physical and chemical parameters (Table 1) such that the experimental results for the flame speed of $\mathrm{CH}_{4}$ /air mixtures in flat burner-stabilized flames $[14,15]$ are reproduced correctly [11].

The equations describing a stationary deflagration process, are the conservation equations for energy, mass fractions, mass and momentum $[3,16]$. The energy and mass fraction equations have the same shape:

$$
\nabla \cdot\left[\rho \mathbf{v} c_{\mathrm{p}} T\right]-\nabla \cdot[\lambda \nabla T]=-\Delta h \dot{\rho}_{\mathrm{fu}}
$$

and

$$
\nabla \cdot\left[\rho v Y_{i}\right]-\nabla \cdot\left[\rho D_{i} \nabla Y_{i}\right]=s_{i} \dot{\rho}_{\mathrm{fu}}
$$

where $\mathbf{v}$ is the velocity vector, $c_{\mathrm{p}}$ the specific heat, $\lambda$

$\dagger \Delta h$ and $c_{\mathrm{p}}$ are calculated in accordance with ref. [17]. $\ddagger$ This definition gives $s_{\mathrm{fu}}=1$.
Table 1. The physical parameters

\begin{tabular}{ccc}
\hline Parameter & Value & Dimension \\
\hline$\rho_{\mathrm{u}}$ & 1.13 & {$\left[\mathrm{~kg} \mathrm{~m}^{-3}\right]$} \\
$T_{\mathrm{u}}$ & 300 & {$[\mathrm{~K}]$} \\
$\lambda$ & $\lambda_{\text {ref }}(T / 1500)^{\gamma}$ & {$\left[\mathrm{J} \mathrm{m}^{-1} \mathrm{~K}^{-1} \mathrm{~s}^{-1}\right]$} \\
$\lambda_{\text {ref }}$ & 0.092 & {$\left[\mathrm{~J} \mathrm{~m}^{-1} \mathbf{K}^{-1} \mathrm{~s}^{-1}\right]$} \\
$\gamma$ & 0.77 & {$\left[\mathrm{Jg}^{-1} \mathrm{~K}^{-1}\right]$} \\
$c_{\mathrm{p}}$ & 1365 & {$\left[\mathrm{~J} \mathrm{~kg}^{-1}\right]$} \\
$\Delta h$ & $4.813 \times 10^{7}$ & {$\left[\mathrm{~kg}_{\mathrm{ox}} \mathbf{k g}_{\mathrm{fu}}^{-1}\right]$} \\
$s_{\mathrm{ox}}$ & 3.883 & {$\left[\left(\mathrm{~kg} \mathrm{~m}^{-3}\right)^{(1-\alpha-\beta)}\right]$} \\
$A$ & $2.6 \times 10^{15}$ & \\
$\alpha$ & 1.2 & {$[\mathrm{~K}]$} \\
$\beta$ & 2.8 & \\
$T_{\mathrm{a}}$ & 16900 & \\
\hline
\end{tabular}

the thermal conductivity, $D_{i}$ the mass diffusion coefficient of species $i$ in the mixture, $\Delta h$ the amount of enthalpy produced by the reaction $\dagger$ and $s_{i}$ the mass of species $i$, consumed per unit mass of fuel. $\ddagger$

Adding (3) for all species (including the inerts and products) should lead to the continuity equation:

$$
\nabla \cdot \rho \mathbf{v}=0,
$$

since the diffusion fluxes and chemical source terms add to zero by definition.

In this paper we will study the behavior of the thermal boundary layer using a flat velocity profile and supposing that the lateral velocity component is equal to zero. Therefore, the flow field is assumed to be one-dimensional :

$$
\nabla \cdot \rho \mathbf{v}=\frac{\partial \rho u}{\partial x}=0
$$

which reduces the calculation of the flow field to one equation:

$$
\rho(x, y) u(x, y)=\rho^{\mathrm{u}} u^{\mathrm{u}}
$$

where the superscript $\mathrm{u}$ denotes the 'unburnt' conditions for $x \rightarrow-\infty$.

An equation of state has to be added to complete the set of equations. The ideal gas law may be used here :

$$
P=\rho R_{\mathrm{g}} T
$$

where $P$ is the pressure and $R_{\mathrm{g}}$ the specific gas constant of the mixture. Most stationary deflagration processes are low Mach number flows, therefore, the pressure $P$ may be assumed to be constant. Furthermore, $R_{\mathrm{g}}$ is assumed to be independent of the mixture composition. Combination of these approximations gives a density depending on temperature only:

$$
\frac{\rho}{\rho^{\mathrm{u}}}=\frac{T^{\mathrm{u}}}{T} \text {. }
$$

The specific heat and the transport coefficients are chosen in such a way that an analytical analysis is applicable. The thermal conductivity is taken to be constant and equal to the thermal conductivity of nitrogen at $T=1500 \mathrm{~K}$. The mass diffusion 
coefficients of fuel and oxygen $\left(D_{\mathrm{fin}}\right.$ and $\left.D_{\mathrm{ox}}\right)$ are chosen in such a way that the Lewis numbers:

$$
L e_{\mathrm{fu}, 0 \mathrm{x}}=\begin{gathered}
\lambda \\
c_{\mathrm{p}} \rho D_{\mathrm{fu}, \mathrm{ox}}
\end{gathered}
$$

are equal to one.

The combined use of the continuity equation (4) and the equation of state (5) makes it possible to eliminate $\rho$ and $u$, thus reducing the set of variables $\left(T, Y_{\mathrm{fu}}\right.$ and $\left.Y_{\mathrm{ox}}\right)$ and conservation equations (2) and (3) to three, coupled by the reaction rate (1).

\section{SCHEMATIC VIEW}

The profiles of temperature, mass fractions and Shvab-Zel'dovich variables

$$
J_{\mathrm{fu}, 0 \mathrm{x}} \equiv \frac{c_{\mathrm{p}} T}{\Delta h}+\frac{Y_{\mathrm{fu}, \mathrm{ox}}}{s_{\mathrm{fu}, \mathrm{ox}}}
$$

look schematically as shown in Fig. 3 as a function of the distance from the burner wall $y$. The flame is stabilized for $y>\delta_{\mathrm{y}}$, whereas the cooling by the burner wall causes the flame to extinguish for $y<\delta_{q}$. Both the temperature and the mass fractions behave as in an undisturbed flat flame at distances from the wall larger than $\delta_{\mathrm{q}}$.

We may expect a decrease of the temperature in the region $0<y<\delta_{\mathrm{q}}$. There is no significant fuel mass consumption in this region, therefore, the mass fractions change only due to diffusion. This means that they will be almost constant over a small distance $\eta$ from the burner wall, since the diffusion flux through the wall is zero. Furthermore, the assumption of Lewis numbers equal to one imposes that the $J_{\text {fu,ox }}$-profiles are not influenced by the presence of the flame [11]. Therefore, the decline of the mass fraction profiles in the intermediate region $\left(\eta<y<\delta_{\mathrm{q}}\right.$ ) has to be proportional to the incline of the temperature profiles, in such a way that $J_{\text {fu.ox }}$ is (almost) constant in this region.

This means that, in the region $\eta<y<\delta_{\mathrm{q}}$, the heat loss by cooling is compensated by a chemical enthalpy

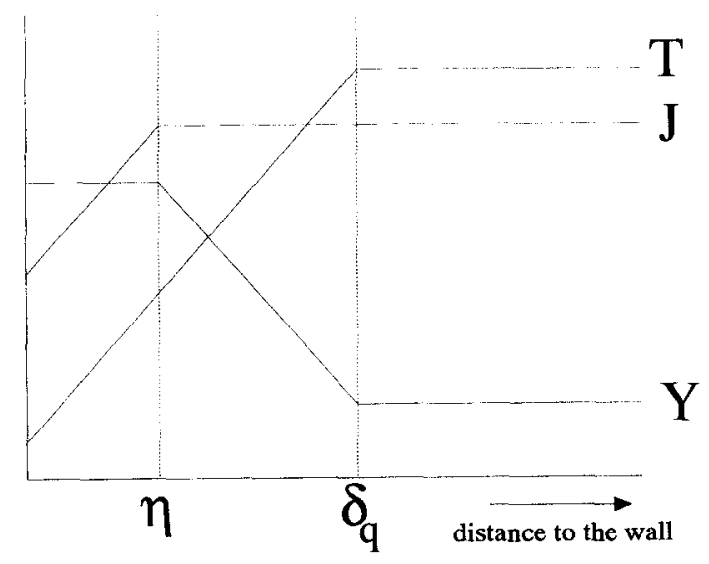

FIG. 3. Schematic profiles for $T, Y_{i}$ and $J_{i}$. flux, caused by diffusion of fuel and oxygen while the heat loss is not compensated in the region $y<\eta$. The above considerations lead to the conclusion that the Shvab-Zel'dovich variables are merely influenced near the wall $(y<\eta)$ and that they have an almost constant value through the remainder of the domain (for all $x<0$ and $y>\eta$ ).

\section{THE THERMAL BOUNDARY LAYER}

An approximate solution of the energy conservation equation with approximate boundary conditions for the thermal boundary layer will be presented in this section. The assumption with respect to the velocity profiles given in Section 2 , combined with a constant thermal conductivity and specific heat leads to the following reduced form of the energy equation (2):

$$
\frac{1}{L} \frac{\partial T}{\partial x}-\frac{\partial^{2} T}{\partial x^{2}}-\frac{\partial^{2} T}{\partial y^{2}}=\Delta h \dot{\rho}_{\mathrm{lu}}
$$

where $L$ equals the length scale defined by:

$$
L=\frac{\lambda}{\rho^{\mathrm{u}} u^{\mathrm{u}} c_{\mathrm{p}}}=\frac{0.092}{1.13 \times 0.4 \times 1365} \simeq 1.5 \times 10^{-4} \mathrm{~m}
$$

As sketched in Fig. 3, the upstream domain of the flame is divided into two regions, as far as the temperature is concerned. There is an undisturbed region $\left(y>\delta_{4}\right)$, where the profiles are independent of the distance to the burner wall (the lateral conduction may be neglected) and a boundary layer $\left(y<\delta_{4}\right)$. where the cooling is important. The flat flame in the undisturbed region is considered to be infinitely thin and positioned at $x=0$. This gives rise to an exponential temperature profile as a function of $x$ :

$$
T(x, y)=T^{u}+\left(T^{b}-T^{u}\right) \mathrm{e}^{x^{\prime} l} \quad \text { for } y>\delta_{\mathrm{q}} .
$$

This leads to the following boundary conditions for the temperature in the cooling layer :

$$
\begin{gathered}
y=0: \quad T=T^{\mathrm{u}}, \\
y \rightarrow \delta_{\mathrm{q}}: T \rightarrow T^{\mathrm{u}}+\left(T^{\mathrm{b}}-T^{\mathrm{u}}\right) \mathrm{e}^{\mathrm{x} / L}
\end{gathered}
$$

and

$$
x \rightarrow-\infty: T=T^{u} .
$$

The closing boundary condition (at $x=0$ ) must be chosen in such a way that it meets the other boundary conditions and the energy equation.

The cooling in the thermal boundary layer is assumed to cause a temperature drop so fast that there is effectively no combustion in this region $\left(\dot{\rho}_{\mathrm{fu}}=0\right)$. By doing so, the shape of the boundary conditions and energy equation (6) imply that a separation of directions might be applied:

$$
T(x, y)=T^{u}+t_{1}(x) t_{2}(y) .
$$

Note that this will give an exact solution of the energy equation under the assumed boundary conditions. Further note, that the assumption of a constant ver- 
tical velocity at inflow $\left(u^{u}\right)$ is essential to come to the disconnection of directions.

In the thermal boundary layer the separation of directions gives :

$$
\frac{1}{t_{1}}\left[\frac{1}{L} \frac{\partial t_{1}}{\partial x}-\frac{\partial^{2} t_{1}}{\partial x^{2}}\right]=\frac{1}{t_{2}}\left[\frac{\partial^{2} t_{2}}{\partial y^{2}}\right] .
$$

The left- and right-hand sides of equation (8) depend only on $x$ and $y$, respectively. This means that both sides have to be equal to a constant $c^{2}$, since the solution has to hold for all values of $x$ and $y$ in the boundary layer. Three different solutions are possible :

$$
\begin{gathered}
\text { for } c^{2}=0: t_{1}=k_{1} \mathrm{e}^{x / L} \text { and } t_{2}=k_{2} y \\
\text { for } c^{2}>0: t_{1}=k_{3} \mathrm{e}^{\Gamma_{ \pm} x} \text { and } t_{2}=k_{4} \mathrm{e}^{c y}+k_{5} \mathrm{e}^{-c y}
\end{gathered}
$$

and

$$
\begin{aligned}
& \text { for } c^{2}\left(=-l^{2}\right)<0: t_{1}=k_{6} \mathrm{e}^{\Gamma_{ \pm} x} \\
& \text { and } t_{2}=k_{7} \sin (l y)+k_{8} \cos (-l y)
\end{aligned}
$$

with

$$
\Gamma_{ \pm}=\frac{1}{2 L}\left[1 \pm \sqrt{ }\left(1-[2 c L]^{2}\right)\right]
$$

The parameters $k_{1, \ldots, 8}$ are constants. In the solutions for $c^{2}<0$ only $\Gamma_{+}$is allowed, since $\Gamma_{-}$is negative and, therefore, gives indefinite results for $x \rightarrow-\infty$. In this study we fully exclude the oscillatory solutions for $c^{2}<0$ in the $y$-direction, since they disturb the one-dimensional flat-flame solution for $y>\delta_{\mathrm{q}}$ as demanded by the boundary condition. Note that the solutions given in equations (9)-(11) also hold for the mass fractions and the Shvab-Zel'dovich variables as long as they are described by the same type of conservation equation and have appropriate boundary conditions.

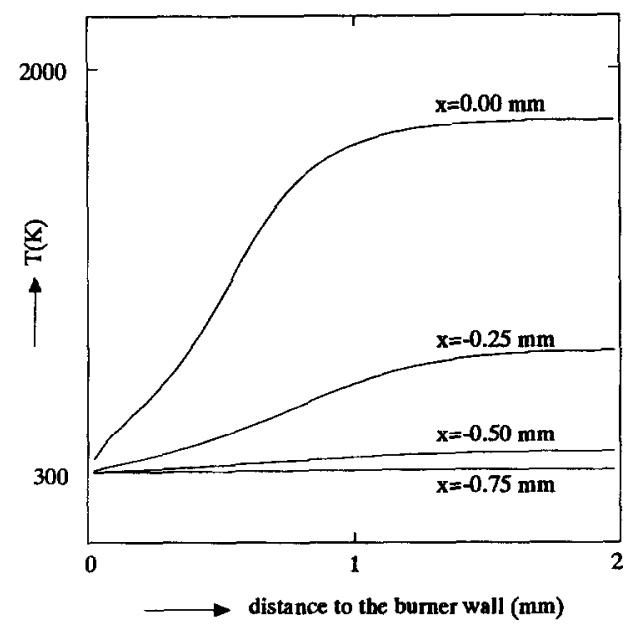

The solution found in (9) meets all boundary conditions for the energy equation (6). It reads :

$$
T(x, y)=T^{\mathrm{u}}+\left(T^{\mathrm{b}}-T^{\mathrm{u}}\right) \frac{y}{\delta_{\mathrm{q}}} \mathrm{e}^{x / L} \quad \text { for } 0<y<\delta_{\mathrm{q}} .
$$

Note that the derivative is discontinuous at $y=\delta_{\mathrm{q}}$. This is a consequence of the presence of the infinitely thin reaction zone at $x=0$. However, the absence of a flame for $x<0$ should give rise to a smooth transition from the linear to the constant behavior near $y=\delta_{\mathrm{q}}$, as in the numerical results (see Fig. 4). The consequence of (12) is that the temperature increases linearly in the $y$-direction for all $y<\delta_{\mathrm{q}}$. From a physical point of view this is easy to understand, for there is only conduction (and no convection) in that direction. It is clear that the assumption of separation of directions in fact provides the additional boundary condition.

The solution in (12) is the result of the disconnection of the fluxes in both directions and, therefore, of the assumed boundary conditions. The expression is confronted with the numerical result in Fig. 4, to verify the validity of these approximations. It shows the same type of behavior as predicted, namely a linear increase of the logarithm of $\left(T-T^{\mathrm{u}}\right) /\left(T^{\mathrm{b}}-T^{\mathrm{u}}\right)$ in $x$-direction and a linear increase of $T$ in $y$-direction. The disconnection of fluxes and the choice of boundary conditions seems, therefore, to be justified. The only unknown in (12) is the standoff distance $\delta_{\mathrm{q}}$. Its size determines the distance of the flame from the burner. A first guess for the value of $\delta_{\mathrm{q}}$ can be made by comparing Figs. 3 and 4 . This gives

$$
\delta_{\mathrm{q}} \simeq 9 \times 10^{-4} \mathrm{~m} \text {. }
$$

\section{THE DIFFUSIVE BOUNDARY LAYER}

We now turn to the calculation of the mass fraction profiles. They obey the same type of reduced con-

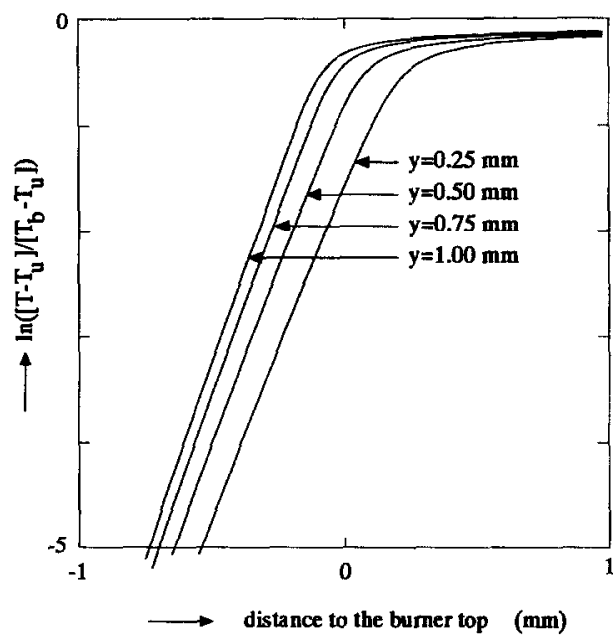

FIG. 4. Numerically determined temperature profiles in $y$-direction $T$ (left) and in $x$-direction $\ln \left(\left(T-T^{\mathrm{u}}\right) /\left(T^{\mathrm{b}}-T^{\mathrm{u}}\right)\right)$ (right). 
servation equation as the temperature (6) following from (3):

$$
\frac{1 \partial Y_{i}}{L} \frac{\partial^{2} Y_{i}}{\partial x}-\frac{\partial^{2} Y_{i}}{\partial y^{2}}=s_{i} \dot{\rho}_{\mathrm{qu}}
$$

where the Lewis numbers have been assumed to be equal to one. $\dot{\rho}_{\mathrm{fu}}$ is again assumed to be equal to zero in the thermal boundary layer $\left(y<\delta_{4}\right)$. This leads to the same set of solutions as presented in (9)-(11). There is, however, a change in the type of boundary condition at the burner wall, since no particles move in or out of the wall. $\partial Y_{i} / \partial y$ has to be equal to zero at $y=0$ for $x<0$. The boundary conditions for $Y_{i}$ are, therefore, given by:

$$
\begin{gathered}
y=0: \frac{\partial Y_{i}}{\partial y}=0 \\
y \rightarrow \delta_{\mathrm{q}}: \quad Y_{i} \rightarrow Y_{i}^{\mathrm{u}}+\left[Y_{i}^{b}-Y_{i}^{\mathrm{u}}\right] \mathrm{e}^{\mathrm{x}^{\prime / l}} \\
x \rightarrow-\infty: \quad Y_{i}=Y_{i}^{\mathrm{u}} .
\end{gathered}
$$

The boundary condition for the mass fractions at the burner wall is responsible for the fact that a solution analogous to (12) is not sufficient to represent the correct behavior of the mass fraction profiles near the wall. The correct shape of the mass fraction profiles is found by the addition of solutions of the $c^{2}>0$ type in (10) to a solution of the $c^{2}=0$ type in (9). Note that the inclusion of the solution of the $c^{2}=0$ type is necessary to ensure that the Shvab-Zel'dovich variables are not influenced by the presence of a flame at $y=\delta_{\mathrm{q}}$. Therefore, the solution of the mass fractions is given by:

$$
Y_{i}(x, y)=Y_{i}^{\mathrm{u}}+\frac{Y_{i}^{\mathrm{b}}-Y_{i}^{\mathrm{u}}}{\partial_{\mathrm{q}}}\left[y \mathrm{e}^{x / L}+\tilde{Y}(x, y)\right]
$$

where $\tilde{Y}(x, y)$ reads :

$$
\begin{aligned}
& \tilde{Y}(x, y)=\int_{0}^{x} \mathrm{~d} c \mathrm{e}^{-c y}\left[F(c) \mathrm{e}^{\left[1+\sqrt{3}\left(1-[2 c L]^{3}\right] x / 2 L\right.}\right. \\
& \left.+G(c) \mathrm{e}^{\left.\left[1-\gamma\left(1-\cdots[2 c l]^{2}\right)\right] x_{i}^{\prime} 2 l\right]}\right] .
\end{aligned}
$$

The amplitudes $F(c)$ and $G(c)$ indicate the contribution of solutions with length scale $1 / c$ in the $y$-direction. Note that solutions with $c<0$ do not contribute since they lead to diverging solutions for $y \rightarrow \infty$. The boundary condition at $y=0$;

$$
\left.\frac{\partial Y_{i}}{\partial y}\right|_{f x, m}=0
$$

will determine $F(c)$ and $G(c)$. The use of solution (14) on this boundary leads to:

$$
\mathrm{e}^{x / L}=\int_{0}^{\infty} c \mathrm{~d} c\left[F(c) \mathrm{e}^{\left[1+\sqrt{3}\left(1-[2 c L]^{2}\right)\right] x i 2 L}\right.
$$$$
\left.+G(c) \mathrm{e}^{\left[1 \cdots \cdot\left(1-[2 c t]^{2}\right\}\right] x i 2 L}\right]
$$

The integral is rewritten by defining:

$$
1 k \equiv \sqrt{ }\left(1-[2 c L]^{2}\right)
$$

for $c>1 / 2 L$ (using $i^{2}=-1$ ). The solutions for which $c<1 / 2 L$ are discarded because the solutions for $c \geqslant 1 / 2 L$ form a complete orthogonal set of functions, sufficient to fulfill (16). We divide (16) by $\mathrm{e}^{\mathrm{x} 2 t}$ and replace $x / 2 L$ by $X$ for the sake of notation. This gives:

$$
\frac{1}{4 L^{2}} \int_{0}^{x} k \mathrm{~d} k\left[F^{\prime}(k) \mathrm{e}^{i k x}+G^{\prime}(k) \mathrm{e}^{i k x}\right]=\mathrm{e}^{x}
$$

where $F^{\prime}(k)$ and $C^{\prime}(k)$ are the amplitudes of the solution $k$ corresponding to $F(c)$ and $G(c)$, respectively. The two integrals may be put together as:

$$
\frac{1}{4 L^{2}} \int_{-\infty}^{x}|k| \mathrm{d} k R(k) \mathrm{c}^{k \cdot k}=\mathrm{e}^{x}
$$

where

$$
R(k)=F^{\prime}(k) \text { for } k>0
$$

and

$$
R(k)=G^{\prime}(-k) \quad \text { for } k<0 .
$$

A Fourier transformation may be applied if the righthand side of (17) is continued as a bounded function for all $x$ values instead of only for values $X<0$. Wc have chosen to do so by means of symmetrical continuation according to:

$$
\text { for } X<0: \frac{1}{4 L^{2}} \int^{d}|k| \mathrm{d} k R(k) \mathrm{e}^{i k X}=\mathrm{e}^{x}
$$

and

$$
\text { for } X>0: \frac{1}{4 L^{2}} \int^{x}|k| \mathrm{d} k R(k) \mathrm{e}^{\mathrm{k} k}=\mathrm{e}^{x} .
$$

Note that different continuations for $X>0$ will lead to different amplitudes $R(k)$. However, they all give rise to the same shape of the profile for $X<0$. The chosen (symmetrical) continuation implies

$$
F(k)=G^{\prime}(-k)
$$

or

$$
R(k)=R(-k) .
$$

Application of the inverse Fourier-transformation now leads to:

$$
\begin{aligned}
\frac{2 \pi}{4 L^{2}}|k| R(k) & =\left[\int_{0}^{0} \mathrm{~d} X \mathrm{e}^{l^{1}|k| X}+\int_{0}^{\infty} \mathrm{d} X \mathrm{e}^{[1+k k\}}\right] \\
& =\frac{2}{1+k^{2}} .
\end{aligned}
$$

Substitution of this solution for $R(k)$ in $\widehat{Y}(x, y)$ gives : 


$$
\begin{aligned}
\tilde{Y}(x, y)= & -\frac{2 L}{\pi} \int_{0}^{\infty} \mathrm{d} k\left[\frac{1}{\sqrt{ }\left(1+k^{2}\right)}\right]^{3} \\
& \times \mathrm{e}^{-\sqrt{(}\left(1+k^{2}\right) y / 2 L} \cos \left(\frac{k x}{2 L}\right) \mathrm{e}^{x / 2 L} \\
= & -\frac{2 L}{\pi} \mathrm{e}^{(x-y) / 2 L} \int_{0}^{\infty} \mathrm{d} k\left[\frac{1}{\sqrt{\left(1+k^{2}\right)}}\right]^{3} \\
& \times \mathrm{e}^{-\left(\sqrt{ }\left(1+k^{2}\right)-1\right) y / 2 L} \cos \left(\frac{k x}{2 L}\right) .
\end{aligned}
$$

Both the factors $\left(1+k^{2}\right)^{-3 / 2}$ and $\mathrm{e}^{-\left(\sqrt{ }\left(1+k^{2}\right)-1\right) y / 2 L}$ indicate that the most important contribution to $\tilde{Y}$ is given by the $k \simeq 0$ values, especially for larger $y$ values. It may therefore be expected from (18) that $\tilde{Y}(x, y)$ has a typical decrease of $\mathrm{e}^{-y / 2 L}$ in the $y$-direction. The term $\widetilde{Y}$ represents the deviation of the mass fraction profiles from their linear incline. The length scale $\eta$ introduced in Section 3 will therefore have a value of the order of $\eta \simeq 2 L \simeq 3 \times 10^{-4} \mathrm{~m}$.

The solution of (14) combined with (18) results in mass fractions at $y=\delta_{\mathrm{q}}$, which are smaller than $Y_{i}^{\mathrm{b}}$. This is physically inappropriate, especially when $Y_{i}^{\mathrm{b}}$ equals zero. Therefore, the boundary condition at $y=\delta_{\mathrm{q}}$ Inust be redefined, which is done by replacing $\left[Y_{i}^{\mathrm{b}}-Y_{i}^{\mathrm{u}}\right] / \delta_{\mathrm{q}}$ in (14) by $\Delta Y_{i} / \delta_{\mathrm{q}}$. Next, $\Delta Y_{i}$ can be found by assuming that the mass fractions are equal to $Y_{i}^{\mathrm{b}}$ in the flame-attachment point $\left(0, \delta_{\mathrm{q}}\right)$ :

$$
\begin{gathered}
Y_{i}\left(0, \delta_{\mathrm{q}}\right)=Y_{i}^{\mathrm{b}}=Y_{l}^{\mathrm{u}} \\
-\frac{\Delta Y_{\mathrm{i}}}{\delta_{\mathrm{q}}}\left\{\delta_{\mathrm{q}}+\frac{2 L}{\pi} \int_{0}^{\infty} \mathrm{d} k\left[\frac{1}{\sqrt{\left(1+k^{2}\right)}}\right]^{3} \mathrm{e}^{-\sqrt{ }\left(1+k^{2}\right) \delta_{\mathrm{q}} / 2 L}\right\},
\end{gathered}
$$

which leads to:

$$
\Delta Y_{i}=\frac{Y_{i}^{\mathrm{u}}-Y_{i}^{\mathrm{b}}}{1+\frac{2 L}{\pi \delta_{\mathrm{q}}} \int_{0}^{\infty} \mathrm{d} k\left[\frac{1}{\sqrt{\left(1+k^{2}\right)}}\right]^{3} \mathrm{e}^{-\sqrt{ }\left(1+k^{2}\right) \delta_{\mathrm{q}} / 2 L}} .
$$

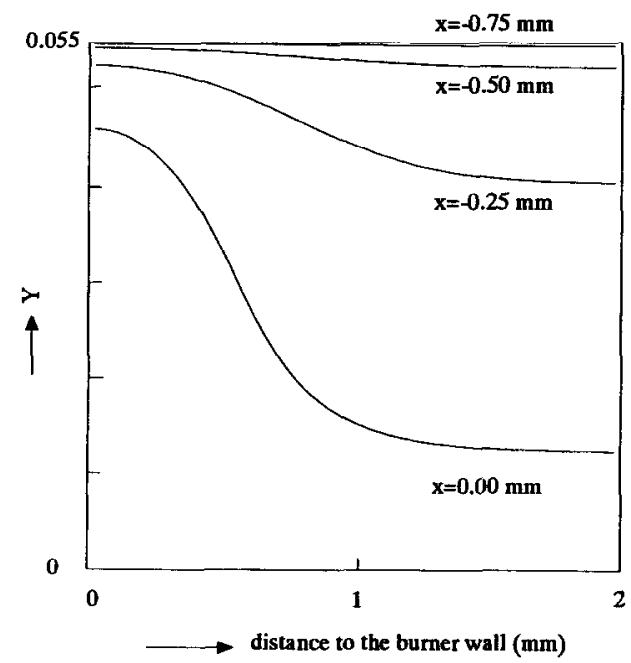

The numerical solution $Y_{\mathrm{fu}}$ of (13) is presented in Fig. 5. It shows that the mass fraction profiles indeed behave as predicted by (14). The exponential decrease in the $x$-dircetion is shown by means of the logarithm :

$$
\ln \left(\frac{Y_{\mathrm{fu}}-Y_{\mathrm{fu}}^{\mathrm{u}}}{Y_{\mathrm{fu}}^{\mathrm{b}}-Y_{\mathrm{fu}}^{\mathrm{u}}}\right)
$$

Note the resemblance of Figs. 4 and 5. The horizontal (no-flux) zone near to the wall is followed by an almost linear decrease with a decline proportional to the increase of temperature in the $y$-direction. Therefore, the disconnection of directions seems to be justified again. Furthermore, the region in which the mass fraction profiles are constant has a width of a few tenths of millimeters, which agrees with the above result for $\eta$.

\section{THE SOLUTION OF THE SHVAB-ZEL'DOVICH VARIABLES}

The expression for $T(x, y)$ in (12) has to be reconsidered before we turn to the solution of the ShvabZel'dovich variables. The combined solutions of the temperature and the mass fractions may not give rise to a discontinuous derivative in the Shvab-Zel'dovich variables at the $y=\delta_{\mathrm{q}}$ boundary, since the flame has no influence on its conservation equations. Therefore, the linear incline of the temperature has to be proportional to the decline of the mass fractions. This gives a new solution $T(x, y)$ :

$$
T(x, y)=T^{\mathrm{u}}+\Delta T \frac{y}{\delta_{\mathrm{q}}} \mathrm{e}^{x / L}
$$

with

$$
\Delta T=\frac{T^{\mathrm{b}}-T^{\mathrm{u}}}{1+\frac{2 L}{\pi \delta_{\mathrm{q}}} \int_{0}^{\infty} \mathrm{d} k\left[\frac{1}{\sqrt{\left(1+k^{2}\right)}}\right]^{3} \mathrm{e}^{-\sqrt{ }\left(1+k^{2}\right) \delta_{q} / 2 L}}
$$

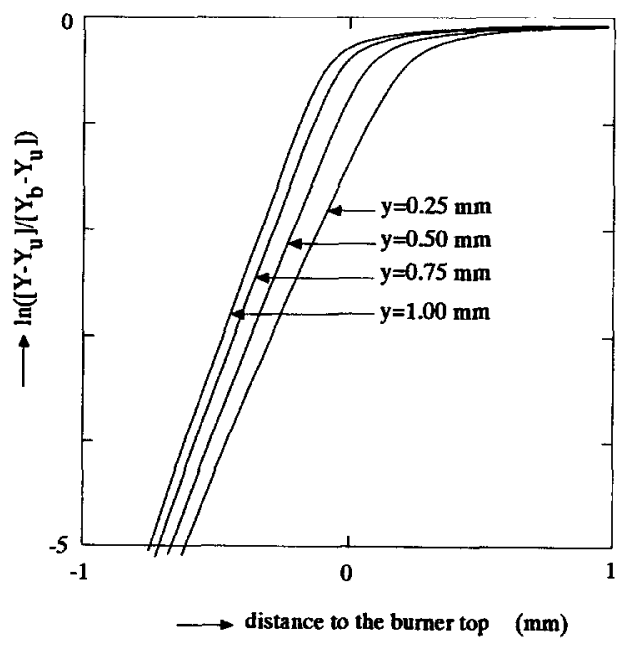

FIG. 5. Numerically determined fuel mass-fraction profiles in $y$-direction $Y_{\mathrm{fu}}$ (left) and in $x$-direction $\ln \left(\left(Y_{\mathrm{fu}}-Y_{\mathrm{fu}}^{\mathrm{u}}\right) /\left(Y_{\mathrm{fu}}^{\mathrm{b}}-Y_{\mathrm{fu}}^{\mathrm{u}}\right)\right)$ (right). 

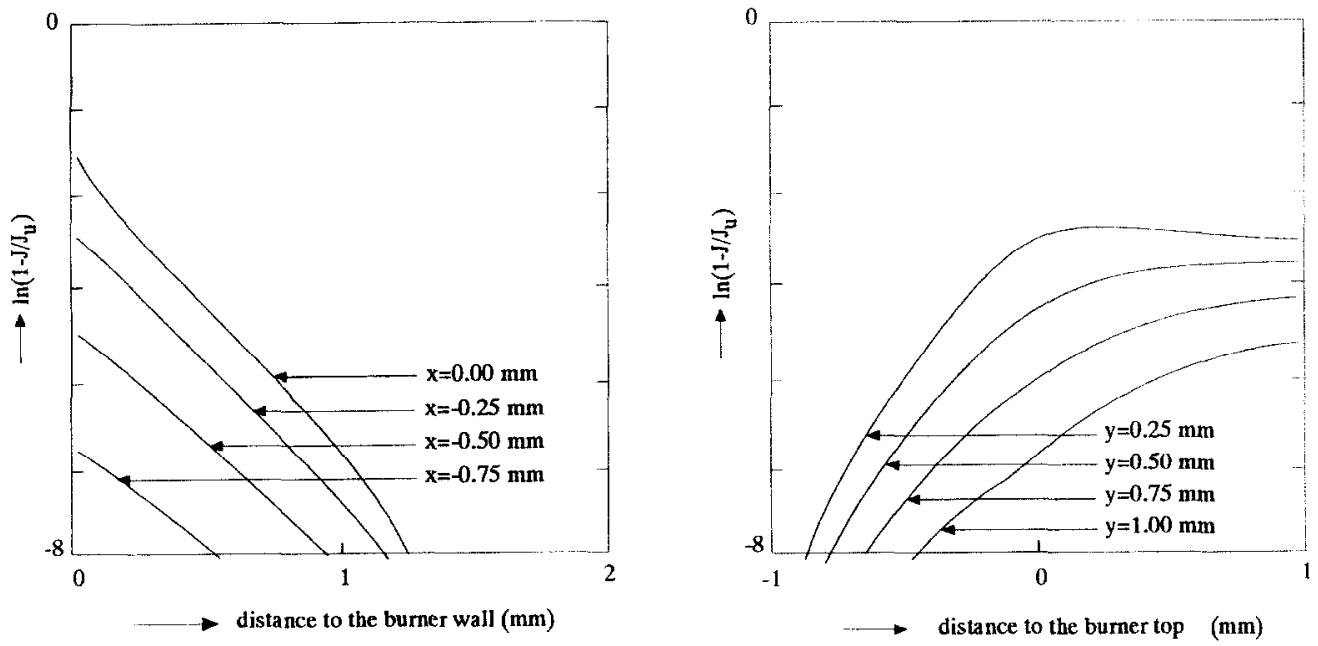

Fig. 6. Numerically determined profiles for the logarithm of the Shvab-Zel'dovich variables for fuel in $y$-direction (left) and in $x$-direction (right).

instead of $T^{\mathrm{b}}-T^{\mathrm{u}}$. Adding $c_{\mathrm{p}} T / \Delta h$ in (20) to $Y_{i} / s_{i}$ in (14), (18) and (19) gives:

$$
\begin{aligned}
& J_{i}(x, y)=J_{i}^{\mathrm{u}}-\frac{2 L \Delta Y_{i}}{\pi} s_{i} \delta_{\mathrm{q}} \mathrm{e}^{(x-y) \cdot 2 L} \int_{0}^{\sigma} \mathrm{d} k\left[\frac{1}{\sqrt{ }\left(1+k^{2}\right)}\right]^{3}
\end{aligned}
$$

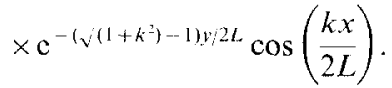

Note that the terms linear in $y$ in the solutions for $T$ and $Y_{i}$ are cancelled, which means that the solution for the Shvab-Zel'dovich variables is indeed not influenced by the presence of the flame at $y=\delta_{\mathrm{u}}$.

The difference in the boundary conditions for the temperature and the mass fractions are the cause of the decrease of $J_{\text {fu, ox }}$ near the burner wall. Therefore, this solution satisfies the conservation equation:

$$
\frac{1}{L} \frac{\partial J_{i}}{\partial x}-\frac{\partial^{2} J_{i}}{\partial x^{2}}-\frac{\partial^{2} J_{i}}{\partial y^{2}}=0
$$

for all values of $y$ (thus also for $y>\delta_{4}$ ), as well as the boundary conditions :

$$
\begin{aligned}
y=0: & \frac{\partial J_{i}}{\partial y}=\frac{\partial \tau}{\partial y} \\
y \gg \eta: & J_{i} \rightarrow J_{i}^{\mathrm{u}} \\
x \rightarrow-\infty: & J_{i}=J_{i}^{\mathrm{u}} .
\end{aligned}
$$

The starting-point of this model is the existence of an unperturbed flame for $y>\delta_{\mathrm{u}}$. As the solution in (21) shows, there is in fact no $y$-value for which the ShvabZel'dovich variables are not influenced by cooling. However, the exponential decrease provides a strongly decreasing influence of the cooling for $y \gg 2 L$. Therefore, $\delta_{\mathrm{q}}$ will have to be larger than $2 L(\simeq \eta)$.

The numerical results for the Shvab-Zel'dovich variable $J_{\text {fu }}$ are shown in Fig. 6, by means of the logarithm of $1-J_{\mathrm{fu}} / J_{\mathrm{fu}}^{\mathrm{u}}$. The logarithm may be expected to be linearly shaped in both directions $x$ and $y$, if the $k \simeq 0$ terms give the most important contributions to $J_{\mathrm{fu}}$ (in accordance with the prediction made on the basis of (18)). This is indeed seen clearly. Therefore, the added result of temperature and mass fractions in the Shvab-Zel'dovich variable (21) proves to be a good approximation of the numerical result. Furthermore, this shows that the loss of enthalpy is well described by the proposed model.

Figure 6 indicates that the length scale:

$$
\eta^{\prime}=-\left[\frac{\partial \ln \left(J_{i}^{\mathrm{u}}-J_{i}\right)}{\partial y}\right]
$$

is a measure for the width of the region of constant mass fraction profiles. $\eta^{\prime}$ determined according to (22) leads to a quantitative estimate for $\eta$. The numerical result for $\eta^{\prime}$ presented in Fig. 7 shows a value of

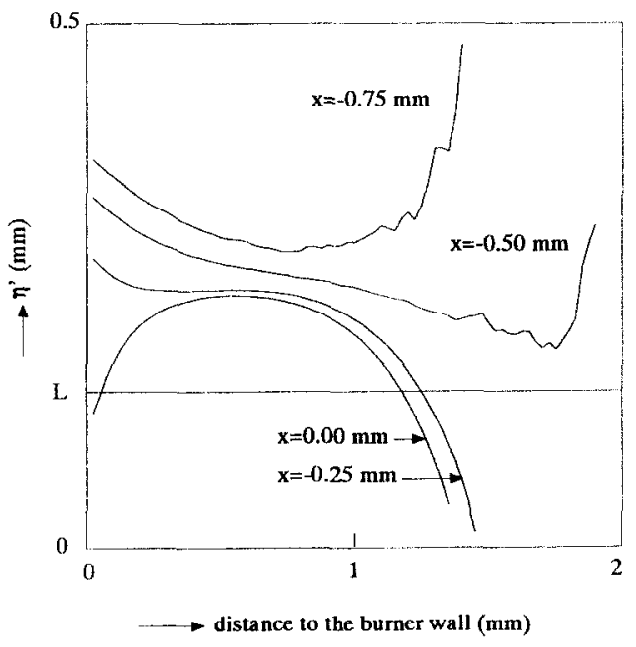

FIG. 7. $\eta^{\prime}$ according to (22). 
approximately $2.3 \times 10^{-4} \mathrm{~m}$, which agrees reasonably well with the estimated value for $\eta$ of $2 L$, found in Section 5 .

\section{THE ENERGY BALANCE}

The solution of the Shvab-Zel'dovich variables will now be used to check the energy balance in the region below the flame. First, the cooling rate $\left(Q_{w}\right)$ by the wall is calculated by using (12):

$Q_{\mathrm{w}}=\left.\int_{-\infty}^{0} \mathrm{~d} x \lambda \frac{\partial T}{\partial y}\right|_{x, y=0}=\frac{\lambda \Delta T}{\delta_{\mathrm{q}}} \int_{-\infty}^{0} \mathrm{~d} x \mathrm{e}^{x / L}=\frac{\lambda \Delta \mathrm{T}}{\delta_{\mathrm{q}}} L$.

The total loss of energy $\left(Q_{\mathrm{r}}\right)$ at the outflow of the burner $(x=0)$ is determined from :

$$
Q_{\mathrm{r}}=\rho^{\mathrm{u}} u^{\mathrm{u}} \Delta h \int_{0}^{\infty} \mathrm{d} y\left[J_{i}^{u}-J_{i}(0, y)\right] .
$$

The use of $J_{i}(0, y)$ according to (21) gives:

$$
\begin{aligned}
Q_{\mathrm{r}} & =\rho^{\mathrm{u}} u^{\mathrm{u}} \Delta h \frac{\Delta Y_{i}}{s_{i} \delta_{\mathrm{q}}} \frac{2 L}{\pi} \int_{0}^{\infty} \mathrm{d} y \int_{0}^{\infty} \mathrm{d} k\left[\frac{1}{\sqrt{ }\left(1+k^{2}\right)}\right]^{3} \\
& \times \mathrm{e}^{\sqrt{ }\left(1+k^{2}\right) y / 2 L} \\
& =\rho^{\mathrm{u}} u^{u} c_{\mathrm{p}} \frac{\Delta T}{\delta_{\mathrm{q}}} \frac{4 L^{2}}{\pi} \int_{0}^{\infty} \mathrm{d} k\left[\frac{1}{\sqrt{ }\left(1+k^{2}\right)}\right]^{4} \\
& =\frac{\rho^{\mathrm{u}} u^{\mathrm{u}} c_{\mathrm{p}}}{\lambda} \lambda \frac{\Delta T}{\delta_{\mathrm{q}}} L^{2}=\frac{\lambda \Delta T}{\delta_{\mathrm{q}}} L,
\end{aligned}
$$

where we used:

$$
\Delta h \frac{\Delta Y_{i}}{s_{i} \delta_{\mathrm{q}}}=c_{\mathrm{p}} \frac{\Delta T}{\delta_{\mathrm{q}}} \text { and } L=\frac{\lambda}{\rho^{\mathrm{u}} u^{\mathrm{a}} c_{\mathrm{p}}} .
$$

The fact that (23) and (24) lead to the same result means that the solution found for the Shvab-Zel'dovich variables indeed satisfies the conservation equations.

It is possible to determine an estimated value for $\delta_{\mathrm{q}}$ by calculating $Q_{\mathrm{w}}$ numerically. However, to obtain an accurate value for $\delta_{\mathrm{q}}$ from equation (23), it is necessary that the flame is stabilized exactly at the height of the top of the burner rim $(x=0)$ and that it is infinitely thin. The numerically found $Q_{w}$ is very sensitive to changes of flame position in the $x$-direction. Our numerical results give a $\delta_{\mathrm{q}}$ of approximately $7 \times 10^{-4}$ $\mathrm{m}$, which agrees reasonably with the estimated value, presented in Section 4.

\section{THE THICKNESS $\delta_{\mathrm{q}}$ OF THE THERMAL BOUNDARY LAYER}

The solution of the conservation equations for energy and species in the thermal boundary layer

$+T\left(0, \delta_{\mathrm{q}}\right)<T^{\mathrm{b}}$ implies that the flame stabilizes with a small angle relative to the $y$-axis.

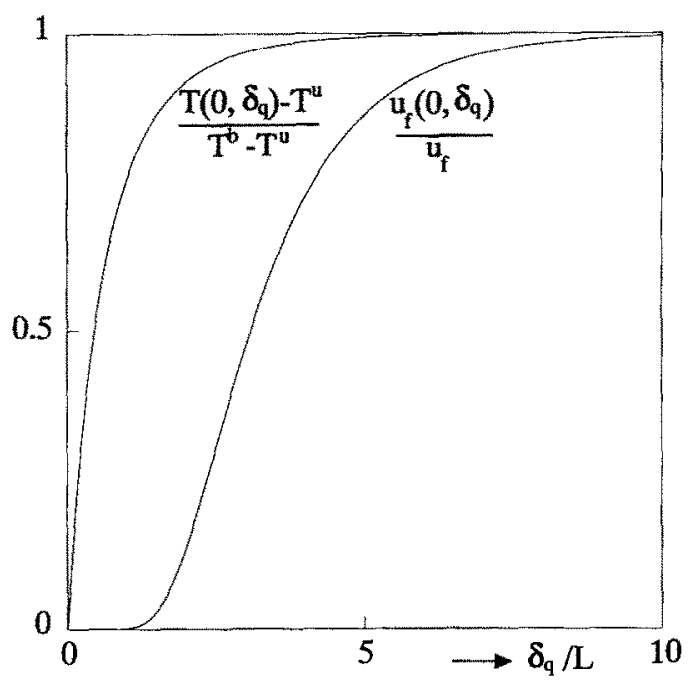

FIG. 8. The temperature and the flame speed in the attachment point $\delta_{q}$ according to (25) and (26), respectively.

$\left(0<y<\delta_{\mathrm{q}}\right)$ and in the region $(0<y<\eta)$ has provided us with a typical length scale $(\eta)$ of the order of $2 L=3 \times 10^{-4} \mathrm{~m}$. This agrees reasonably well with the value for $\eta^{\prime}$ determined according to (22), which gives $\eta^{\prime} \simeq 2.3 \times 10^{-4} \mathrm{~m}$. The model also implies that $\eta$ is a minimum value for $\delta_{4}$. The temperature profiles calculated numerically, support this conclusion. They lead to an estimated value for $\delta_{\mathrm{q}}$ of order $9 \times 10^{-4} \mathrm{~m}$.

The numerical values for the stand-off distance, presented above, are in fact the consequence of the numerical balance between the local values of the flame speed and the gas speed. It is also possible to derive a value for $\delta_{\mathrm{q}}$ on the basis of an analytical analysis of that balance. This is done as follows. The flame stabilizes in the point $(x, y)=\left(0, \delta_{\mathrm{q}}\right)$, where the flame speed reaches the gas speed (which has been chosen to be equal to the adiabatic flame speed). However, the solution of (21) indicates that the adiabatic flame speed and maximum temperature $T^{b}$ are reached only for $y \rightarrow \infty$. This means that $\delta_{\mathrm{q}}$ has to be large enough, so that the temperature and the flame speed are close to their adiabatic values in the attachment point. $f$ An equation for the temperature in the attachment point $T\left(0, \delta_{\mathrm{q}}\right)$ is found by using (20):

$$
\begin{aligned}
\frac{T^{\mathrm{b}}-T^{\mathrm{u}}}{T\left(0, \delta_{\mathrm{q}}\right)-T^{\mathrm{u}}}=1+\frac{2 L}{\pi \delta_{\mathrm{q}}} \int_{0}^{\infty} \mathrm{d} k & {\left[\frac{1}{\sqrt{ }\left(1+k^{2}\right)}\right]^{3} } \\
& \times \mathrm{e}^{-\sqrt{ }\left(1+k^{2}\right) \delta_{\mathrm{q}} / 2 L}
\end{aligned}
$$

The flame speed in the attachment point $\left(u_{i}\left(0, \delta_{q}\right)\right)$ relative to the adiabatic value $\left(u_{s}\right)$ is calculated by using the analytical relation between the flame temperature and the flame speed of a flat burner-stabilized methane/air flame $[11,18]$. This results in:

$$
\ln \left(\frac{u_{\mathrm{f}}\left(0, \delta_{\mathrm{q}}\right)}{u_{\mathrm{f}}}\right)=\frac{-T_{\mathrm{a}}}{2 T\left(0, \delta_{\mathrm{q}}\right)}+\frac{T_{\mathrm{a}}}{2 T^{\mathrm{b}}}+\frac{p+2}{2} \ln \left(\frac{T\left(0, \delta_{\mathrm{q}}\right)}{T^{\mathrm{b}}}\right)
$$


for a stoichiometric mixture. The results of (25) and (26) are shown in Fig. 8 as a function of $\delta_{\mathrm{y}} / L$. It is clear from these figures that $\delta_{\mathrm{q}} / L$ must have a value close to (or larger than) 5 to obtain a flame speed in the attachment point close to the adiabatic value. The resulting value for $\delta_{4} \simeq 8 \times 10^{+} \mathrm{m}$ agrees with previous estimates.

The above analysis indicates that the value for $\delta_{1}$ is influenced mainly by the length scale $L$ and by the ratio $T_{\mathrm{r}} / T^{\mathrm{b}}$.

\section{DISCUSSION}

In this section we will first discuss the influence of the choice of a constant thermal conductivity and a constant velocity profile within the thermal boundary layer. Subsequently, the results found for $\delta_{\mathrm{q}}$ will be compared with some experimental data found in the literature.

So far, we assumed that $i$ has a constant value (equal to the value for nitrogen at $T=1500 \mathrm{~K}$ ). In reality, the thermal conductivity is temperature dependent and varies significantly through a flame. The presented analytical description clearly shows the influence of the chosen value for the thermal conductivity (through $L$ ) on $\delta_{4}$. It may, therefore, be expected that the temperature dependence of the thermal conductivity also influences the value found for $\delta_{4}$. Numerical results on the structure of the boundary layers using a more realistic model for the thermal conductivity are presented in Appendix A. These results indicate that the values for $\eta^{\prime}$ and $\delta_{4}$ do not change considerably, although some changes in the shape of the profiles are observed.

The influence of changes in the velocity profile is studied to some extent in Appendix B. The results show that the shape of the temperature and mass fraction profiles are clearly influenced by the chosen velocity profile, especially in the near vicinity of the wall $(0<y<\eta)$. However, there appears to be no significant change in the profiles close to the attachment point $\left(y=\delta_{\mathrm{q}}\right)$. Furthermore, the thickness of the thermal boundary layer $\delta_{\mathrm{q}}$ hardly changes.

We now turn to the comparison of the presented values for the stand-off distance $\delta_{\mathrm{y}}$ and experimental results found in the literature. The available data on critical gradients near the flash-back limit will be used to make this comparison. In the definition of critical gradients it is assumed that the thickness of the thermal boundary layer is independent of the shape of the burner (and consequently also of the velocity profile near the burner wall). We adopt this assumption by neglecting this dependence, based on the results of Appendix B. We further assume that the flame stabilizes in the attachment point $\left(0, \delta_{q}\right)$ as long at the gas speed equals $u_{f}$ in this point $[2,3]$. These assumptions lead to:

$$
\delta_{4}=\begin{aligned}
& u_{\mathrm{f}} \\
& g
\end{aligned}
$$

where $g$ is the gradient of the velocity profile in the boundary layer.

The description presented here concentrates on conditions ahead of the flame. These conditions do not change when the flame moves further down in the burner. Consequently, the value of $\delta_{4}$ is equal to its value at the flash-back limit. Thus, $\delta_{\mathrm{c}}$ must be equal to the ratio of the adiabatic flame speed and the critical gradient for flash-back. Harris et al. [19] find a critical gradient for flash-back of $400 \mathrm{~s}^{-1}$ for a stoichiometric methane/air flame on a cylindrical Runsen burner. This gives an estimated value for $\dot{b}_{4}$ of $0.41 / 400 \mathrm{~m} \simeq 10 \times 10^{4} \mathrm{~m}$.

Another point of reference for the value of $\partial_{4}$ is the quench distance, which should comespond roughly with a value of $2 \dot{\delta}_{4}$. For a stoichiometric $\mathrm{CH}_{4}$ air flame von Karman and Millan [6] and Harris et at. [19] find a quench distance of $24 \times 10^{-4} \mathrm{~m}$, experimentally. This leads to $\delta_{\mathrm{q}}=12 \times 10^{-4} \mathrm{~m}$.

The agreement between the experimental and the theoretical results is satisfactory. certainly in view of the mentioned physical simplifications and the indirec way in which the results are compared with cxperimental data.

\section{CONCLUSION}

The given analytical description leads to results for the temperature, mass fractions and Shvab-Zel'dovich variables and a value for $\eta$ which agree very well with the corresponding profiles found numcrically. The analytical treatment ((25) and (26)) shows that the length scale $L$ and the ratio $T_{a} / T$ dominate the thickness of the boundary layers $\delta_{4}$. The comparison with experimental results on flash-back and quench limits shows that the theoretically predicted thickness of the thermal boundary layer agrees reasonably well with experiments.

\section{REFERENCES}

1. G. von Elbe and B. Lewis, Theory of ignition, quenching and stabilization of flames of nonturbulent gas mixtures. Third Symp. on Combustion. Flame and Explosion Phenomena, pp. 6879 (1949).

2. B. Lewis and $\mathrm{G}$. von Elbe, Combustion Flames and Explosions of Gases. Academic Press, New York (1961).

3. R. A. Strehlow, Combustion Fundamentals. McGrawHili, New York (1984).

4. G. Dixon-Lewis, Combustion Chemistry (Edited by W. C. Gardiner). Springer, New York (1984).

5. S. L. Nly and C. E. Hermance, A two-dimensional theory of laminar flame quenching. Combust. Flame 40, 173 185 (1981)

6. T. von Karman and G. Millan, Thermal theory of a laminar flame front near a cold wall, Fourth Symp. (International) on Combustion (The (ombustion Institute), pp. 173177 (1953)

\%. J. D. Buckmaster, The quenching of deflagration waves, Combust. Flame 26, 151-162 (1976).

8. M. Gerstein and W. B. Stine, Analytical criteria for fammability limits, Fourleenth Symp. (International) on Combustion (The Combustion Institute), pp. 1109-1118 (1973). 
9. H. C. de Lange and L. P. H. de Goey, Modeling of twodimensional laminar flames, Symp. on Num. Methods in Thermal Problems, Vol. 7, pp. 1171-1181 (1991).

10. H. C. de Lange and L. P. H. de Goey, Modeling of laminar premixed methane-air fiames of a parallel-plate burner, Symp. on Num. Methods in Thermal Problems, Vol. 7, pp. 1182-1192 (1991).

11. H. C. de Lange, Modelling of laminar premixed gasflames, Ph.D. dissertation, University of Technology Eindhoven (1992).

12. F. A. Williams, Combustion Theory. Addison-Wesley, Massachusetts (1965).

13. J. D. Buckmaster, The Mathematics of Combustion. SIAM, Philadelphia (1985).

14. G. E. Andrews and D. Bradley, The burning velocities of methane-air mixtures, Combust. Flame 19, 275-288 (1972).

15. W. E. Kaskan, Flame temperature and mass buming velocity, Sixth Symp. (International) on Combustion (The Combustion Institute), pp. 134-143 (1967).

16. R. B. Bird, W. E. Stewart and E. N. Lightfoot, Transport Phenomena. Wiley, New York (1960).

17. W. J. Moore, Physical Chemistry. Prentice-Hall, Englewood Cliffs, New Jersey (1972).

18. I. Glassman, Combustion, pp. 64-75. Academic Press, New York (1977).

19. M. E. Harris, J. Grumer, G. von Elbe and B. Lewis, Burning velocities, quenching, and stability data on nonturbulent flames of methane and propane with oxygen and nitrogen, Third Symp. on Combustion Flame and Explosion Phenomena, pp. 80-89 (1949)

20. N. B. Vargaftic, Tables on the Thermophyical Properties of Liquids and Gasses. Wiley, New York (1980).

\section{APPENDIX A. THE THERMAL CONDUCTIVITY ASSUMPTION}

The influence of the assumption that the thermal conductivity is constant, is studied in this appendix. The results of Sections 5-7 are judged by means of a comparison with the results of numerical calculations. This is done by choosing a thermal conductivity according to:

$$
\lambda=\lambda_{\text {ref }}\left[\frac{T}{1500}\right]^{y}
$$

where the value of $\lambda_{\text {ref }}$ is equal to the value, used for $\lambda$ previously and where $\gamma$ is equal to 0.77 . This representation of the thermal conductivity is based on a fit of the temperature dependence of the thermal conductivity of nitrogen according to the experimental data of ref. [20]. The Lewis numbers are still assumed to be equal to one. The results of this calculation (mass fraction profiles, the Shvab-Zel'dovich variable $J_{\text {fu }}$ and $\eta^{\prime}$ ) are compared in Fig. A.l with the results found previously (with $\gamma=0$ ).

It is clear that the mean value for $\eta^{\prime}$ does not change significantly, although its behaviour is somewhat more irregular than before. The value for $\delta_{\mathrm{q}}$ also hardly changes. In retrospect we may say that, as far as the stand-on distance is concerned, the constant value for thermal conductivity, which has been used in the previous sections, is well chosen.

\section{APPENDIX B. THE VELOCITY PROFILE IN THE BOUNDARY LAYER}

The influence of changes in the velocity profile is tested in this appendix by the introduction of an nth-order velocity profile near the wall. This profile is given by:

$$
\text { for } y<y_{0}: u=u_{0}\left[1-\left\{1-\frac{y}{y_{0}}\right\}\right]
$$

and

$$
\text { for } y \geqslant y_{0}: \quad u=u_{0}
$$

with $y_{0}$ equal to $8 \times 10^{-4} \mathrm{~m}$ and $u_{0}$ equal to $0.4 \mathrm{~m} \mathrm{~s}^{-1}$. Note that (B1) is an $n$ th-order velocity profile around $y=y_{0}$. Further note that the limiting situation with $n \rightarrow \infty$ corresponds with a constant velocity profile as used in the previous sections. The horizontal velocity component is again assumed to be equal to zero. In Fig. Bl we compare the
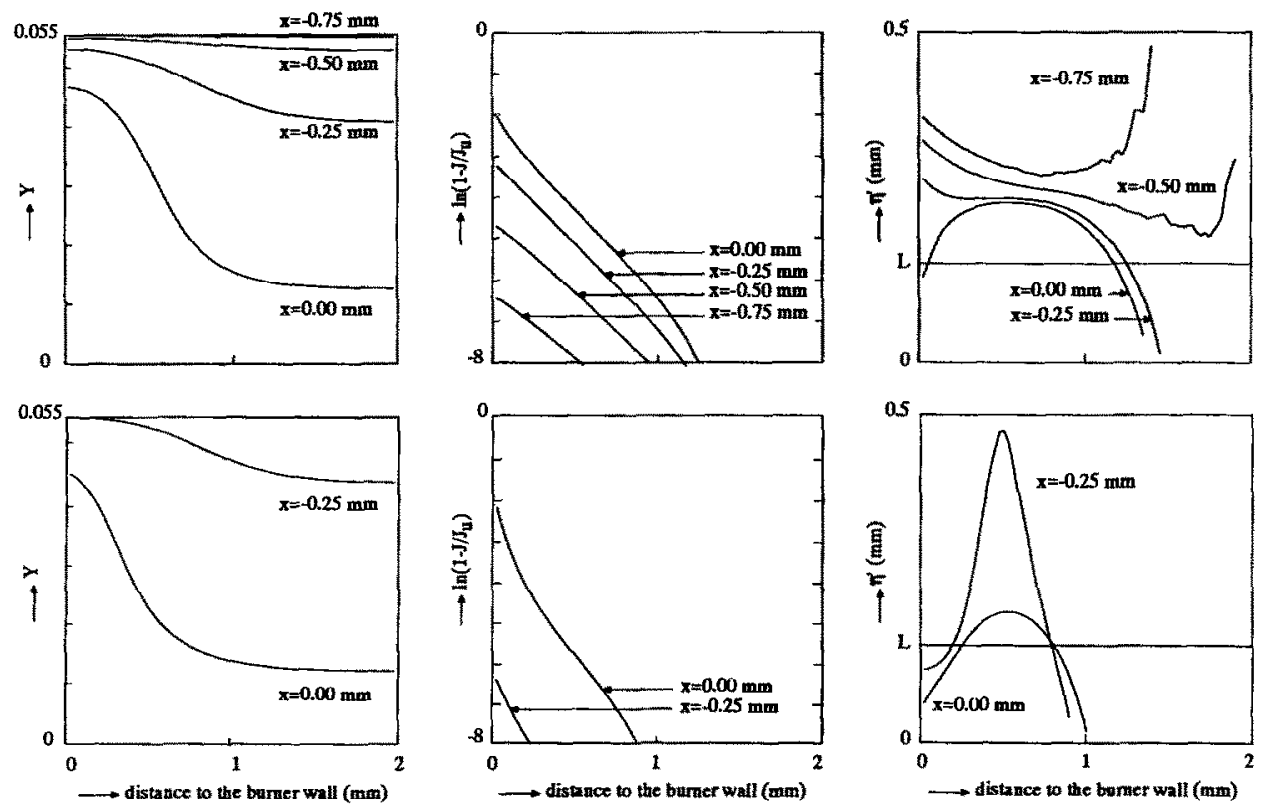

FIG. A1. The profiles in $y$-direction for (from left to right) $Y_{f u}, \ln \left(1-J_{f a} / J_{f u}^{u}\right)$ and $\eta^{\prime}$ according to (22) for $\gamma=0$ (top) and $\gamma=0.77$ (bottom). 

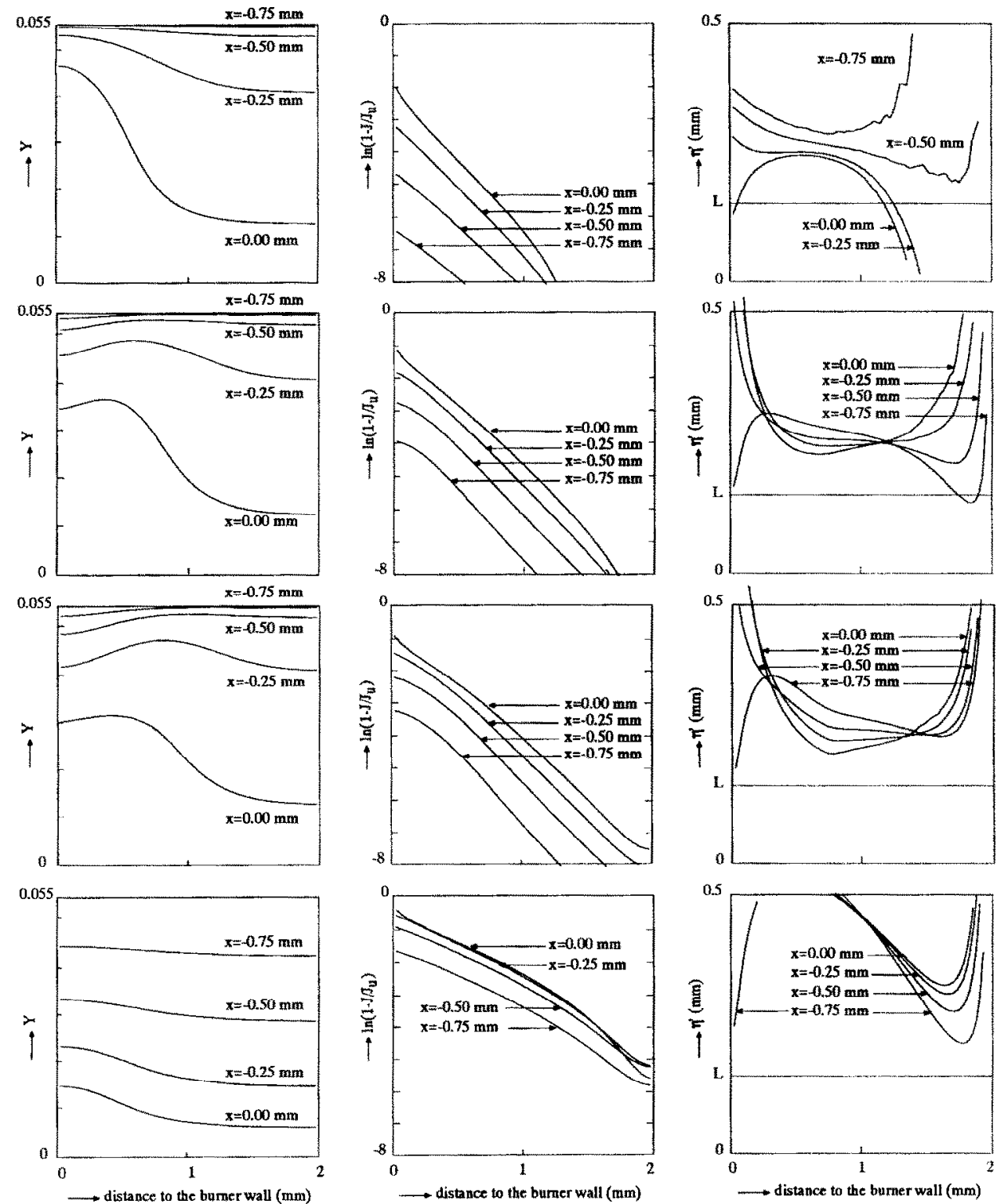

FiG. BI. The profiles in $y$-direction for (from left to right): $Y_{\mathrm{fu}}, \ln \left(1-J_{\mathrm{fu}} / J_{\mathrm{iu}}^{\mathrm{u}}\right)$ and $\eta$ according to (22) using (from top to bottom) : $n \rightarrow \infty, n=2, n=1$ and $n=0$.

profiles of $Y^{14}, J_{t, 1}$ and $\eta^{\prime}$ as a function of $y$ for the velocity profiles with $n=0,1$ and 2 and for $n \rightarrow \infty$, respectively.

Note that the shape of the profiles remains more or less the same in the intermediate region $\eta<y<\delta_{\alpha}$. while large changes are observed in the region $y<\eta$. This is caused by the fact that the velocity of the gas mixture close to the burner is approximately zero, instead of $u_{0}$ when $n \rightarrow \infty$, which results in lower fuel and oxygen mass fractions along the burner wall. The velocity approaches $u_{0}$ somewhat further away from the burner (for all values of $n$ ), thus causing the similarity between the results for the testcd values of $n$ (not equal to 0 ) in the intermediate region. However, the magnitude of $\eta^{\prime}$ and the thickness of the boundary layer $\delta_{4}$ does not change significantly, in spite of the changes in the profiles near the wall. 